\title{
The Titan Environmental Transmission Electron Microscope: Specifications, Considerations and First Results
}

\author{
T.W. Hansen*, J.B.Wagner, * J.R. Jinschek** and R.E. Dunin-Borkowski* \\ * Center for Electron Nanoscopy, Technical University of Denmark, DK-2800 Lyngby, Denmark \\ ** FEI Europe, Achtseweg Noord 5 Bldg. 5651GG Eindhoven, The Netherlands
}

Over the past few decades, the demand for high spatial resolution in situ characterization techniques has increased dramatically. In electron microscopy, this demand constitutes an intrinsic challenge as the electron source requires high vacuum to function. Nevertheless, in the 1970's, transmission electron microscopes (TEMs) were first adapted for use with gases [1]. Such machines are known as environmental transmission electron microscopes or ETEMs and are now in widespread use [2,3]. Although these tools are unique and represent a source of invaluable information, care has to be taken when using them and many additional considerations are required when compared to conventional TEM. In particular the parameter space that affects the result of an experiment increases significantly, and it becomes even more important to consider the effect of both electron/solid and electron/gas interactions.

It is important to remember that ETEM experiments are not carried out under real or operando conditions. Parameters such as reaction rates may therefore be different when measured in an ETEM, especially in catalysis where reactions are often realized at pressures of up to $10^{2}$ bar. Nevertheless, the gap between TEM and true operando conditions has been narrowed significantly. This advance in instrumentation makes it possible to follow dynamic phenomena such as particle formation, nanostructure growth and oxide reduction [4].

The newly installed ETEM at the Center for Electron Nanoscopy at the Technical University of Denmark (DTU) provides a unique combination of techniques for studying materials of interest to the catalytic as well as the electronics and other communities [5]. DTU's ETEM is based on the FEI Titan platform providing ultrahigh microscope stability pushing the imaging resolution into the subÅngström regime. The microscope is equipped with an image spherical aberration $\left(C_{S}\right)$ corrector to reduce the influence of low-order aberrations on imaging, thereby improving image interpretability and minimizing delocalization effects during in situ atomic resolution observations of catalytic reactions. DTU's ETEM has a monochromated field emission electron source and a high-energy resolution post-column energy filter (GIF Tridiem 866) bringing the resolution in electron energyloss spectroscopy (EELS) down to around $200 \mathrm{meV}$. This capability allows EELS fine structure analysis of active catalyst materials as well as of gases using high-energy electrons.

In addition to microscope performance (stability and resolution) the primary challenges of ETEM experiments involve stable and reproducible control of gas pressure, gas flux, and temperature (heating) of gas and specimen. Increased power is required to operate TEM heating holders in the presence of gas in the column as a result of the transport of heat away from the sample region by the gas. Even small variations in gas flow will result in large variations in holder and specimen temperature giving rise to sample drift and instability. DTU's ETEM is equipped with digital mass flow controllers for improving the stability of the gas flow. Unfortunately, the present design of the ETEM results in a significant base pressure in the microscope column (often resulting in sample and gas contamination) as the pumping capacity in the sample region is decreased when switching the microscope from conventional high vacuum mode to ETEM mode. The base pressure is considerably higher in ETEM mode when compared with high vacuum mode, in part because the 
ion getter pumps that reduce the pressure in the sample region down to ca. $10^{-7} \mathrm{mbar}$ (in high vacuum mode) are turned off resulting in a pressure of ca. $10^{-4}$ mbar in ETEM mode. At first sight this limitation might be expected to limit only low-pressure gas/solid reactions in the ETEM $(<0.5$ mbar). However, it also has an impact on higher pressure experiments (5-20 mbar) as it leads to the presence of at least a few atomic layers of contamination on sample surfaces. Great care therefore has to be taken when conducting an ETEM experiment. In addition to the use of high-purity gases and long-term bake-out of the system prior to experimentation, in situ plasma cleaning is carried out to minimize surface contamination and to clean the sample region in general.

Among the first experiments carried out in DTU's ETEM while working on the determination of a perfect ETEM set-up, are high spatial resolution HRTEM studies of gas-solid interactions and high energy-resolution (monochromated) EELS investigations of various gases as shown in Fig. 1. Results obtained from the in situ reduction of catalysts illustrate both sintering phenomena and morphological changes of supported metallic crystals, while EELS studies of different gases are being assessed as a possible means of monitoring gas pressure in the microscope column during ETEM experiments. First results along with general experimental considerations on in situ transmission electron microscopy will be presented.

Jan-Dierk Grunwaldt from DTU Chemical Engineering is greatly acknowledged for providing catalyst samples and suggestions for ETEM experiments.

\section{References}

[1] R.T.K. Baker and P.S. Harris, J. Phys. E Sci. Instrum. 5 (1972) 793.

[2] E.D. Boyes and P.L. Gai, Ultramicrosopy 67 (1997) 219.

[3] P.L. Hansen et al., Adv. Catal. 50 (2006) 77.

[4] A.K. Datye, J. Catal. 216 (2003) 144.

[5] S. Hofmann et al., Nature Materials, 7 (2008) 372.
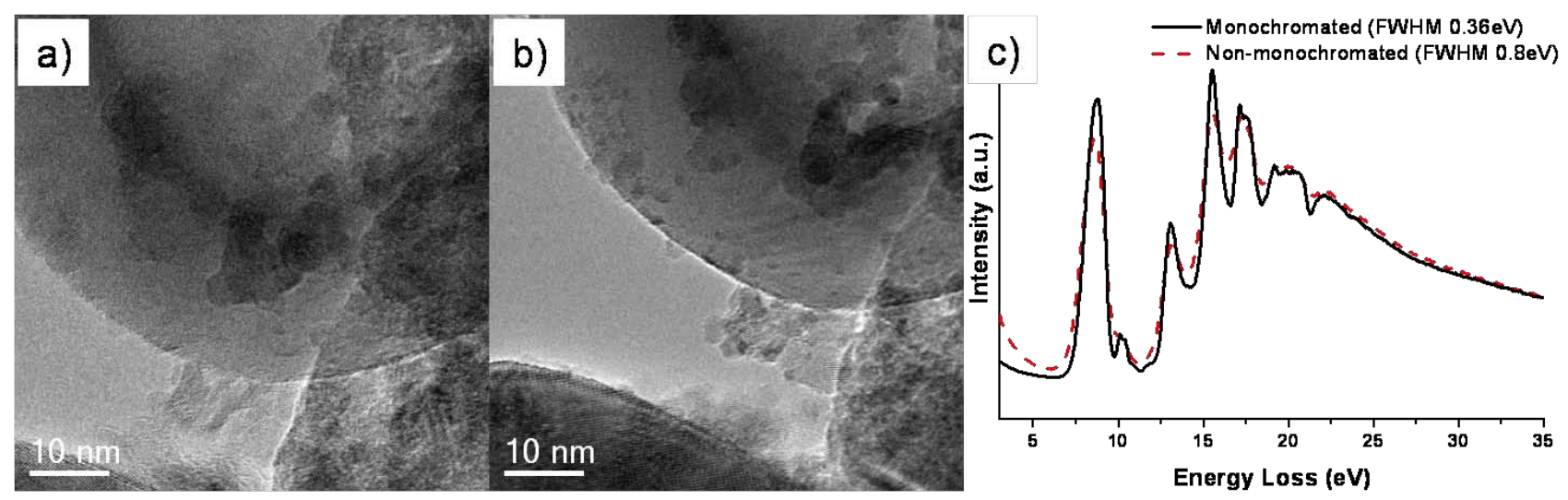

FIG. 1. a) HRTEM image of an as-prepared $\mathrm{Pd} / \mathrm{ZrO}_{2}$ catalyst acquired in high-vacuum at room temperature. b) The same area imaged in $1.3 \mathrm{mbar}_{2}$ at $200^{\circ} \mathrm{C}$. c) Monochromated and nonmonochromated electron energy-loss spectra acquired from 5 mbar molecular oxygen. 